\title{
Efficacy of Cryotherapy Application on the Pain Perception during Intraoral Injection: A Randomized Controlled Trial
}

\author{
Lakshimi Lakshmanan¹, Vignesh Ravindran ${ }^{2}$
}

\begin{abstract}
Background: Profound local anesthetic delivery promotes successful treatment for children in terms of easing their fear, anxiety, and discomfort during dental procedures. Local anesthetic injections are the utmost anticipated or anxious stimuli in the dental operatory. Precooling the oral mucosa by application of cryotherapy before local anesthetic injections can alter the pain perception in children.

Aim and objectives: To compare the efficacy of cryotherapy application and $20 \%$ benzocaine gel at reducing pain perception during buccal infiltration in pediatric patients.

Materials and methods: In this split-mouth study, 30 pediatric patients between 7 years and 10 years of age who needed maxillary buccal infiltration of local anesthetics bilaterally for dental treatment were selected. They received cryotherapy (ice pack) on one quadrant (test group) for 2 minutes and $20 \%$ benzocaine topical gel on the contralateral quadrant (control group). A pediatric dentist blinded to the study assessed sound, eye, motor (SEM) scale based on patients' reaction during injection (objective method), and patients were instructed to use a visual analog scale (VAS) to rate their distress during injection (subjective method). Statistical analyses were performed using Wilcoxon and Mann-Whitney $U$ tests. Results: The cryotherapy group had significantly reduced pain scores on the VAS scale $(40.66 \pm 14.60)$ when compared with the topical anesthetic gel group $(61.33 \pm 9.73)$. The cryotherapy group had reduced pain scores on the SEM scale as well $(1.2 \pm 0.1)$ when compared with the topical anesthetic gel group $(1.6 \pm 0.1)$, which was not statistically significant.
\end{abstract}

Conclusion: When compared with topical anesthetic gel, precooling the injection site with cryotherapy is beneficial in reducing pain before local anesthesia injection in pediatric patients.

Clinical significance: Cryotherapy application eliminates the fear of pain ascribed to injection of local anesthesia and assists in providing pertinent dental care.

Keywords: Cryotherapy, Local anesthesia injection, Pain, Topical anesthesia.

International Journal of Clinical Pediatric Dentistry (2021): 10.5005/jp-journals-10005-2032

\section{INTRODUCTION}

Pain is contemplated to be the intention for pursuing dental services, yet it is also observed to be the vital cause for ignoring it. A local anesthetic injection is one of the potent techniques to alleviate pain, but injection of local anesthetic itself is a major source of patient fear. ${ }^{1}$ Administering local anesthesia to pediatric patients seems to be the most challenging factor in pediatric dental practice. The sensation of injection or needle imports an adverse psychological impact on the child. Insufficient anesthesia exacerbates the discomfort associated with needles, potentially hindering proper dental treatment. ${ }^{2}$

Researchers have carried out various desensitization methods such as the application of topical anesthetics, warming and buffering the anesthetic solution, modifying the rate of flow of infiltration, counter-irritation, vibration or pressure, acupuncture, hypnosis, computer-controlled delivery system (WAND), using appliances like vibraject, dental vibe, or jet injectors to minimize the pain experienced during the administration of local anesthetic agents. $^{3-12}$

Over the years, topical anesthetics are used consistently in dentistry to prepare injection sites before needle penetration. Some studies have proved topical anesthetic capability in reducing pain perception during injections; however, others have concluded that topical anesthetics afford little more than placebo levels of effectiveness. ${ }^{13,14}$ Cryotherapy application is another endorsed strategy for reducing pain perception in patients that would
1,2Department of Pediatric and Preventive Dentistry, Saveetha Dental College and Hospital, Chennai, Tamil Nadu, India

Corresponding Author: Lakshimi Lakshmanan, Department of Pediatric and Preventive Dentistry, Saveetha Dental College and Hospital, Chennai, Tamil Nadu, India, Phone: +91 8939346341, e-mail: lachulaxmanan@gmail.com

How to cite this article: Lakshmanan L, Ravindran V. Efficacy of Cryotherapy Application on the Pain Perception during Intraoral Injection: A Randomized Controlled Trial 2021;14(5):616-620.

Source of support: Nil

Conflict of interest: None

be effective, efficient, and cost-effective. Cryoanesthesia is the application of ice or refrigerant spray on the anesthetic site to counter nerve conduction of pain from the site. ${ }^{15}$ Studies have reported that it lowers edema, nerve conduction velocities, cellular metabolism, and local blood flow. ${ }^{2,16}$ The application of ice provides a physiological as well as psychological benefit to the patients as it may distract them from focusing on their discomfort. ${ }^{17}$ Sprains, wounds, fractures, bruises, insect stings, and other physical injuries have all been managed with this procedure. Their use is much less widespread in dentistry; nevertheless, the use of ice sticks, refrigerants, or vapocoolants in the dental operatory to provide pre-injection anesthesia has been described in the literature. ${ }^{18}$ 
As a further investigation into the efficacy of application of a coolant, this study compares it to a $20 \%$ benzocaine topical anesthetic gel during the administration of buccal infiltration in pediatric patients.

\section{Materials and Methods}

This split-mouth randomized clinical trial was conducted in a dental institute. The ethical approval for the study was obtained from the Institutional Review Board in accordance with the Helsinki Declaration of 1975 (IHEC/SDC-PEDO1803/19/035). Before treatment, informed consent was obtained from all the parents/guardians. Based on data from a previously published article, ${ }^{18}$ the sample size of 30 was obtained with $95 \%$ power using a power analysis.

A total of 57 subjects between 7 years and 10 years of age were recruited for the study. They were subjected to initial clinical examination. The inclusion criteria were: (1) children were healthy (ASA I); (2) required maxillary buccal infiltration anesthesia for dental procedures (extraction, crown preparation, pulp therapy) in both the quadrants; (3) had no prior history of dental treatment; (4) exhibited Frankl's behavior rating grade III or IV, and (5) were not taking any analgesics or other drugs that would influence their perception of pain. Exclusion criteria were children with (1) history of the medically compromised condition; (2) any allergy to local anesthesia; (3) active pathology at the site of injection; (4) prior history of dental treatment; (5) exhibited Frankl's behavior rating grade I or II. Based on the exclusion and inclusion criteria, 27 subjects were eliminated from the study and the total study sample was finalized to 30 children. Enrolment and allocation of participants are shown in Flowchart 1.

The prime intervention included in this split-mouth trial was the application of an ice pack. A quadrant of the patient's dental arch obtained the intervention, which included the cryotherapy application while other quadrants of the same patient received the control, topical anesthetic gel ( $20 \%$ benzocaine) in different appointments. Based on a computer-generated randomization method, the side of the maxilla (right or left) and which preinjection anesthesia (ice pack or benzocaine gel) to be used at each appointment were determined. Meantime between the first and second appointments was 2 weeks, to ensure that all symptoms and possible side effects had subsided.

One postgraduate helped in providing the pre-injection anesthesia (ice pack or benzocaine gel) for all the subjects during both their visits. To prepare the ice pack, 30 lidocaine cartridges were emptied and filled with saline, and stored in a freezer. The temperature of the ice pack was between $-4^{\circ} \mathrm{C}$ and $0^{\circ} \mathrm{C}$ to avoid any risk of frostbite. The suggested time for application of ice on the mucosa is $2-5$ minutes. ${ }^{19}$ Five minutes can be abided by adults, but in pediatric patients, it may result in a lack of cooperation. Hence, in this study, the oral mucosa was dried with a cotton roll for 30 seconds, and an ice pack was applied to the buccal mucosa for 2 minutes (Fig. 1). For the side involving topical benzocaine gel (control group), the oral mucosa was dried with a cotton roll for 30 seconds before application of the pre-injection anesthesia. Topical anesthetic benzocaine gel 20\% (Benzocaine, Beutlich, USA) was applied for 1 minute using cotton with the help of a tweezer.

The procedure was followed by infiltration injection of $2 \%$ lidocaine with 1:80,000 adrenaline (LOX* 2\% ADRENALINE) using a short 27-gauge needle (Unilock, Dispovan) by a single operator blinded to the topical pre-injection technique.

Another dentist who was completely unaware of the topical pre-injection method objectively recorded sound, eye, motor (SEM) score during injection from a $1.5-\mathrm{m}$ distance and a visual analog scale (VAS) was used to evaluate the participants' self-reports of injection pain. The participants were asked to mark the line between faces to pinpoint the pain level they experienced and scored accordingly (Fig. 2 and Table 1).

\section{Statistical Analysis}

Data were tabulated and analyzed using SPSS software version 21.0 (SPSS Inc., Chicago, USA). Wilcoxon and Mann-Whitney $U$ tests

Flowchart 1: Enrolment and allocation of study participants

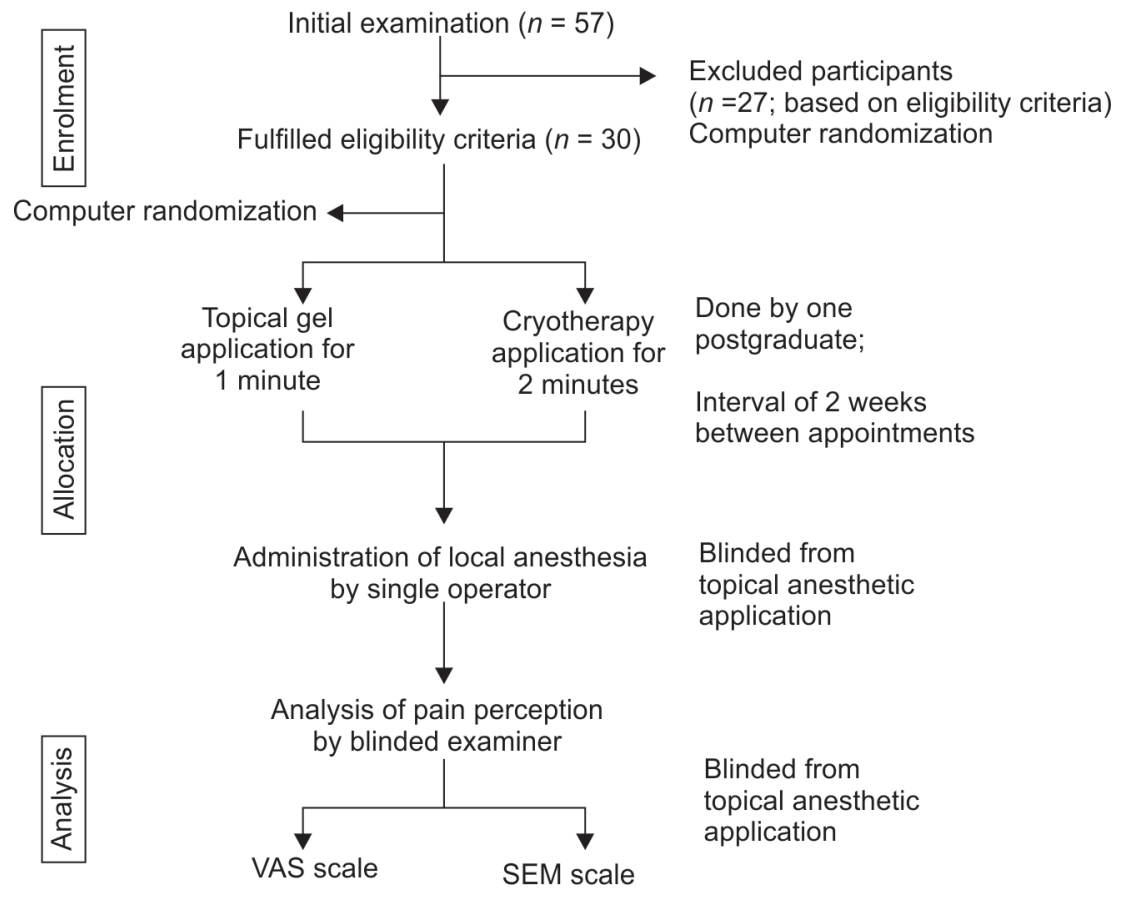



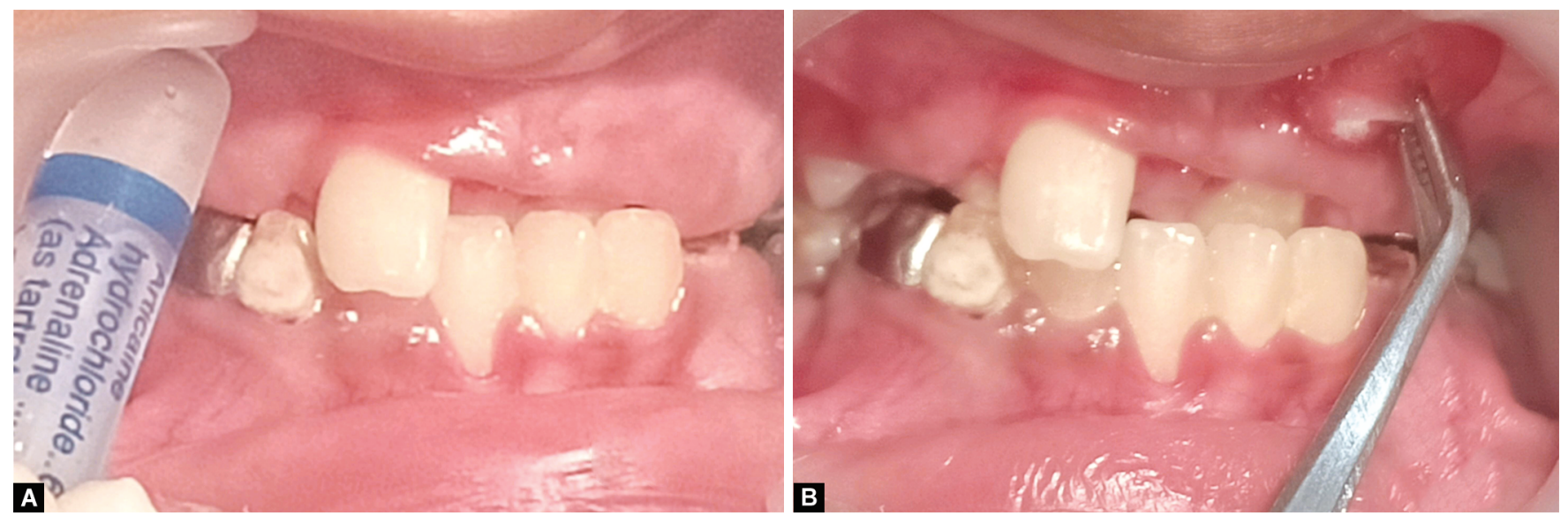

Figs 1 A and B: (A) Application of cryotherapy (ice pack) on the injection site; (B) Application of topical anesthetic gel (benzocaine) on the injection site

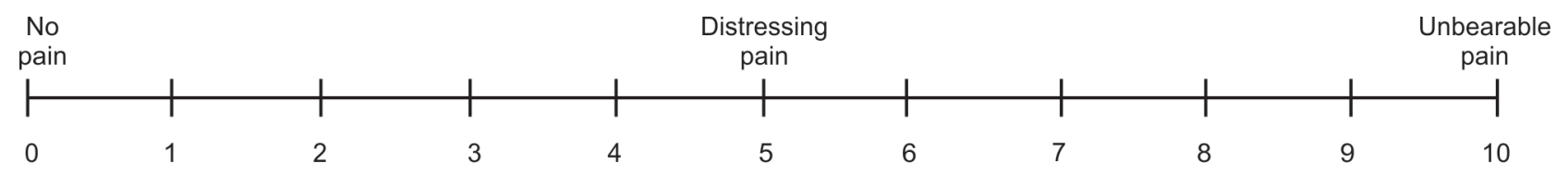

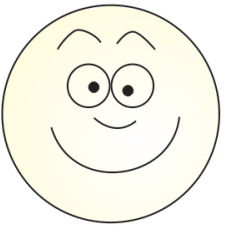

0

No hurt

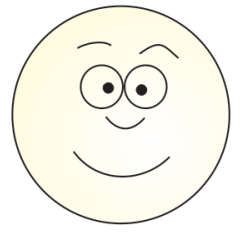

1

Hurts little bit

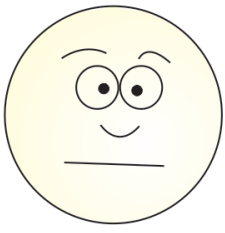

2

Hurts little more

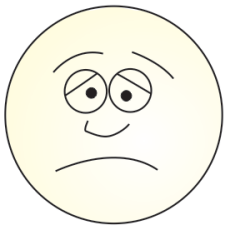

3

Hurts even more

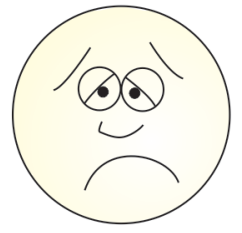

4

Hurts whole lot

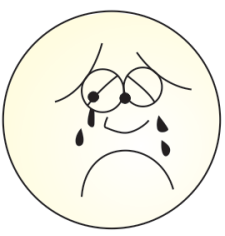

5

Hurts worst

Fig. 2: Sound eye motor (SEM) scale

Table 1: Visual analog scale (VAS)

\begin{tabular}{llll}
\hline Parameter & Comfort & Mild discomfort & Moderate discomfort \\
\hline Sound & No sound & Non-specific sound probable pain & $\begin{array}{l}\text { Verbal complaint, louder discomfort } \\
\text { sound }\end{array}$ \\
Eye & No sign & $\begin{array}{l}\text { Dilated eyes without tears (anxiety complaint, shouting crying } \\
\text { sign) }\end{array}$ & $\begin{array}{l}\text { Tears, sudden eye } \\
\text { movement }\end{array}$ \\
Motor & $\begin{array}{l}\text { Relaxed body and } \\
\text { hand status }\end{array}$ & $\begin{array}{l}\text { Muscular contraction, contraction of } \\
\text { the hand }\end{array}$ & $\begin{array}{l}\text { Sudden body and hand } \\
\text { movements }\end{array}$ \\
\hline
\end{tabular}

were performed for comparison of means. $p<0.05$ determined the statistical significance.

\section{Results}

The participant pool included 17 boys and 13 girls $(N=30)$, ages 7-10 years, with a mean age of $8.28 \pm 0.14$ years.

The means of SEM values for the study and control groups were not statistically significant in total (SEM); whereas sound (S) and motor $(M)$ parameters individually were significantly less for the cryotherapy group $(p<0.05)$ (Table 2$)$.

The mean visual analog score for the study and control groups were $40.66 \pm 14.60$ and $61.33 \pm 9.73$, respectively, with a statistically significant lower score in the cryotherapy group $(p<0.05)$ (Table 3 ).

\section{Discussion}

The most significant challenges that dentists confront are dental anxiety and needle phobia. Local anesthesia is an imperative part of dentistry. The effective treatment of pediatric children by pacifying their fear and distress during dental treatment is aided by the use of a profound local anesthetic. ${ }^{20}$ Various techniques are proposed to lessen the distress of local anesthesia injection amidst which desensitizing the injection site is an endorsed technique. Traditionally, anesthetic gels are preferred as pre-injection topicals. Recognizing the disadvantage of topicals anesthetics, including unpleasant taste, added expense, collateral numbness, and ability to anesthetize minimal thickness of mucosa (1-3 $\mathrm{mm})$, have encouraged to utilize other alternatives. ${ }^{9}$ 
Effectiveness of Cryotherapy on the Pain Perception during Intraoral Injection

Table 2: Comparison of sound eye motor scale scores in cryotherapy and topical gel groups

\begin{tabular}{lllllll}
\hline & \multicolumn{3}{c}{ Cryotherapy } & & \multicolumn{3}{c}{ Topical gel } & \\
\cline { 2 - 3 } SEM & Mean & Standard deviation & & Mean & Standard deviation & p value \\
\hline Sound & 1.0 & 0.8 & 1.4 & 0.9 & $0.04^{*}$ \\
Eye & 1.4 & 0.6 & 1.6 & 0.7 & 0.13 \\
Motor & 1.3 & 0.8 & 1.7 & 0.8 & $0.05^{*}$ \\
Total & 1.2 & 0.1 & 1.6 & 0.1 & $>0.05$ \\
\hline
\end{tabular}

*Statistically significant

Table 3: Comparison of visual analog scale scores in cryotherapy and topical gel groups

\begin{tabular}{llll}
\hline VAS & Mean & Standard deviation & $p$ value \\
\hline Cryotherapy & 40.6 & 14.6 & $<0.001$ \\
Topical gel & 61.3 & 9.7 & \\
\hline
\end{tabular}

*Statistically significant

Literature suggests that cryoanesthesia can be performed to relieve pain from local anesthetic injections, manage postoperative pain, and prevent edema. ${ }^{12}$ Cryoanesthesia acts by stimulating myelinated A-fibers and inhibitory pain pathways, which elevates the pain threshold. It decelerates the nerve conduction, resulting in temporary vasoconstriction. ${ }^{21}$ The main advantage of cryoanesthesia is that it conducts on all the cells of the part and not just on the nerve cells as other topical anesthetics do, thereby generating instantaneous anesthesia. The basis of the analgesic effect of cryotherapy could be explained by "the gate control theory of pain". As the brain can acknowledge only one sensation at a time, cryotherapy when used as a defense to anesthetic injection, reaches the brain before the pain sensation does. Hence, counterstimulation (cryotherapy) reduces pain perception. ${ }^{12}$ Even though the effect produced by cryoanesthesia is only for a brief period, it is ample to reduce the discomfort induced by the insertion of an anesthetic needle. ${ }^{22}$ Cryoanesthesia can be achieved using refrigerant sprays or by application of an ice pack.

The objective of the current trial was to assess the efficacy of cryotherapy on pain perception in pediatric patients and to compare it with topical anesthetic gel (20\% benzocaine). In this splitmouth study, pediatric patients requiring bilateral buccal infiltration for any of the dental procedures were recruited to evade any bias, considering that the pain perception is different for each patient. The results of this study support the rationale that cryotherapy application at the injection site significantly mitigates pain at the time of penetration of needle during delivery of local anesthetic for dental treatment. This was observed in VAS and SEM scales where the scores were less in the cryotherapy group when compared with the topical anesthetic gel group. The results are in accordance with the study conducted by Harbert, who utilized ice to alleviate the discomfort linked with palatal injections. ${ }^{23}$ Comparable findings were reported by Duncan et al. and Hameed et al. who utilized a refrigerant spray as a cooling agent in contact with the tissue before administrating intraoral injection. ${ }^{24,25}$ Aminabadi and Farahani described the efficiency of 2 minutes cryotherapy application before nerve block in reducing the pain perception. ${ }^{19}$ The results of the current study are supported by Kosaraju and Vanderwalle, Kuwahara and Skinner, in different trials, reporting the decrement in pain by application of cryotherapy on the anesthetic site, disregarding their assessment, not including objective scale. ${ }^{26,27}$
Furthermore, a clinical trial by Aminah et al. evaluated the influence of different desensitizing strategies on decreasing injection pain in children, including local anesthetic gel, precooling the injection site, vibration, and buffering the anesthetic solution, and concluded that precooling the injection site considerably reduced the pain perception in children. ${ }^{2}$ In contrast, Wiswall et al. reported that there were no significant VAS differences while comparing pain perception response to pressure, topical anesthetic, and pre-coolant application before palatine nerve block. ${ }^{28}$

The findings of the current trial established the notion that precooling the injection site increases the tolerance to injection while a local anesthetic is being administered and aids in the management of the pediatric patient during dental procedures. The study design had two main advantages: triple blinding and split-mouth design that minimized the risk of bias. However, the limitation of this study includes the rate of injection and needle depth, which were consistent but not identical because of the variable anatomy encountered. Unable to blind the participants, due to the difference in temperature between benzocaine gel and ice pack, and the different methods of application and taste, made it obvious which method was used. The palatal injection which is considered to be more agonizing in comparison to other sites of the oral mucosa has not been included in this study. However, further studies with a larger sample size, which compares other topical agents and local anesthetic techniques, may be warranted.

\section{Conclusion}

Based on the findings of this study, precooling the injection site before local anesthetics might be preferred as an easy and economical auxiliary technique that is beneficial to all pediatric patients with fear and anxiety.

\section{Clinical Significance}

Injection of local anesthetics is a major fear or anxiety-inducing stimuli in the dental operatory. Cryotherapy application eliminates the fear of pain ascribed to injection of local anesthesia and assists in providing pertinent dental care.

\section{References}

1. Chandrashekharan J, Prabhu D, Sunayana M. Efficacy of painless injection technique-vibraject-clinical trial in Chennai, India. Int J Med Dent Sci 2014;3(1):250-255.

2. Aminah $M$, Nagar Priya, Singh $P$, et al. Comparison of topical anesthetic gel, pre-cooling, vibration and buffered local anesthesia on the pain perception of pediatric patients during the administration of local anesthesia in routine dental procedures. Int J Contemp Med Res 2017;4(2):400-403.

3. Colaric KB, Overton DT, Moore K. Pain reduction in lidocaine administration through buffering and warming. Am J Emerg Med 1998;16(4):353-356. DOI: 10.1016/s0735-6757(98)90126-7. 
4. Touyz LZ, Lamontagne $P$, Smith BE. Pain and anxiety reduction using a manual stimulation distraction device when administering local analgesia oro-dental injections: a multi-center clinical investigation. J Clin Dent 2004;15(3):88-92.

5. Hutchins HS, Young FA, Lackland DT, et al. The effectiveness of topical anesthesia and vibration in alleviating the pain of oral injections. Anesth Prog 1997;44(3):87-89.

6. Friedman MJ, Hochman MN. The AMSA injection: a new concept for local anesthesia of maxillary teeth using a computer-controlled injection system. Quintessence Int 1998;29(5):297-303.

7. Halar EM, DeLisa JA, Brozovich FV. Nerve conduction velocity: relationship of skin, subcutaneous and intramuscular temperatures. Arch Phys Med Rehabil 1980;61(5):199-203.

8. Meechan JG, Winter RA. A comparison of topical anesthesia and electronic nerve stimulation for reducing pain of intra-oral injections. Br Dent J 1996;181(9):333-335. DOI: 10.1038/sj.bdj.4809252.

9. Blanton $\mathrm{PL}$, Jeske $\mathrm{AH}$. Dental local anesthetics: alternative delivery methods. J Am Dent Assoc 2003;134(2):228-234. DOI: 10.14219/jada. archive.2003.0140.

10. Vongsavan K, Vongsavan N. Comparison of topical anesthetic gel and TENS in reducing pain. J Dent Res 1996;75:248.

11. Gibson RS, Allen K, Hutless S, et al. The wand vs. traditional injections: a comparison of pain related behaviors. Pediatr Dent 2000;22(6):458462.

12. Shilpapriya M, Jayanthi M, Reddy VN, et al. Effectiveness of new vibration delivery system on pain associated with injection of local anesthesia in children. J Indian Soc Pedod Prev Dent 2015;33(3):173176. DOI: 10.4103/0970-4388.160343.

13. Gill CJ, Orr DL. A double-blind crossover comparison of topical anesthetics. J Am Dent Assoc 1979;98(2):213-214. DOI: 10.14219/jada. archive.1979.0476.

14. Martin MD, Ramsay DS, Whitney $C$, et al. Topical anesthesia: differentiating the pharmacological and psychological contributions to efficacy. Anesth Prog 1994;41(2):40-47.

15. Priyantham $S$, Nuvvula S. Intraoral topical anaesthesia in paediatric dentistry: review. Int J Pharm Bio Sci 2016;7(4):346-353.

16. Ghaderi F, Banakar S, Rostami S. Effect of pre-cooling injection site on pain perception in pediatric dentistry: a randomized clinical trial. Dent Res J 2013;10(6):790-794.
17. Greenstein G. Therapeutic efficacy of cold therapy after intraoral surgical procedures: a literature review. J Periodontol 2007;78(5):790800. DOI: 10.1902/jop.2007.060319.

18. DiMarco AC, O'Kelley Wetmore A. Clinical comparison: fast-acting and traditional topical dental anesthetic. Anesth Prog 2016;63(2):55-61. DOI: 10.2344/0003-3006-63.2.55.

19. Aminabadi NA, Farahani RM. The effect of pre-cooling the injection site on pediatric pain perception during the administration of local anesthesia. J Contemp Dent Pract 2009;10(3):43-50. DOI: 10.5005/ jcdp-10-3-43.

20. Joseph A, Giovannitti JR, Rosenberg MB, et al. Pharmacology of local anesthetics used in oral surgery. Oral Maxillofacial Surg Clin N Am 2013;25(3):453-465. DOI: 10.1016/j.coms.2013.03.003.

21. Hindocha N, Manhem F, Bäckryd E, et al. Ice versus lidocaine $5 \%$ gel for topical anaesthesia of oral mucosa - a randomized cross-over study. BMC Anesthesiol 2019;19(1):227. DOI: 10.1186/s12871-0190902-8.

22. Bleakley C, McDonough S, MacAuley D. The use of ice in the treatment of acute soft tissue injury: a systematic review of randomized controlled trials. Am J Sports Med 2004;32(1):251-261. DOI: $10.1177 / 0363546503260757$.

23. Harbert $\mathrm{H}$. Topical ice: a precursor to palatal injections. J Endod 1989;15(1):27-28. DOI: 10.1016/S0099-2399(89)80094-9.

24. Duncan JD, Reeves GW, Fitchie JG. Technique to diminish discomfort from the palatal injection. J Prosthet Dent 1992;67(6):901-902. DOI: 10.1016/0022-3913(92)90617-j.

25. Hameed NN, Sargod SS, Bhat SS, et al. Effectiveness of precooling the injection site using tetrafluorethane on pain perception in children.J Indian Soc Pedod Prev Dent 2018;36(3):296-300. DOI: 10.4103/JISPPD. JISPPD_222_17.

26. Kosaraju A, Vanderwalle KS. A comparison of a refrigerant and a topical anesthetic gel as pre-inection anesthetics. J Am Dent Assoc 2009;140(1):68-72. DOI: 10.14219/jada.archive.2009.0020.

27. Kuwahara RT, Skinner RB. EMLA versus ice as a topical anesthetic. Dermatol Surg 2001;27(5):495-496. DOI: 10.1097/00042728200102000-00028.

28. Wiswall AT, Bowles WR, Lunos S, et al. Palatal anesthesia: Comparison of four techniques for decreasing injection discomfort. Northwest Dent 2014;93(4):25-29. 